\title{
Heterogeneity in liver histopathology is associated with GSK-3 $\beta$ activity and mitochondrial dysfunction in end-stage diabetic rats on differential diets
}

\author{
Jun-Ho Lee 1,2,\#, Soo-Bong Choi, , Dong-Jun Sung, ${ }^{4, \#}$, Mingli Jin ${ }^{5}$, Ju-Han Lee ${ }^{1}$, Ji-Young Mun ${ }^{6}$, Tae-Sook Hwang ${ }^{7}$, \\ Sang-Don Han ${ }^{8}$, Young-Tae Ro ${ }^{1}$, Sung-Young Kim ${ }^{1}$, Jueng-Soo You ${ }^{1}$, Inja Lim ${ }^{9}$ E Yun-Hee Noh ${ }^{1, *}$ \\ ${ }^{1}$ Department of Biochemistry, Konkuk University School of Medicine, Seoul 05029, ${ }^{2} \mathrm{C} \&$ R Research, Seoul 06199, ${ }^{3}$ Department of Internal \\ Medicine, Konkuk University Chungju Hospital, Konkuk University School of Medicine, Chungju 27376, ${ }^{4}$ Division of Sport and Health \\ Studies, College of Biomedical and Health Science, Konkuk University, Chungju 27478, ${ }^{5}$ Gachon Institute of Pharmaceutical Sciences, \\ College of Pharmacy, Gachon University, Incheon 21936, ${ }^{6}$ Department of Structure and Function of Neural Network, Korea Brain Research \\ Institute, Daegu 41062, ${ }^{7}$ Department of Pathology, Konkuk University School of Medicine, Seoul 05029, ${ }^{8}$ Department of Medical \\ Education, Konkuk University School of Medicine, Seoul 05029, ${ }^{9}$ Department of Physiology, College of Medicine, Chung-Ang University, \\ Seoul 06974, Korea
}

While liver histopathology is heterogeneous in diabetes, the underlying mechanisms remain unclear. We investigated whether glycemic variation resulting from differential diets can induce heterogeneity in diabetic liver and the underlying molecular mechanisms. We generated end-stage non-obese diabetic model rats by subtotal-pancreatectomy in male SpragueDawley rats and ad libitum diet for 7 weeks $(n=33)$. The rats were then divided into three groups, and fed a standard- or a low-protein diet (18 or $6 \mathrm{kcal} \%$, respectively), for another 7 weeks: to maintain hyperglycemia, 11 rats were fed ad libitum (18AL group); to achieve euglycemia, 11 were calorierestricted (18R group), and 11 were both calorie- and proteinrestricted with the low-protein diet (6R group). Overnightfasted liver samples were collected after the differential diets together with sham-control (18S group), and histology and molecular changes were compared. Hyperglycemic-18AL showed glycogenic hepatopathy $(\mathrm{GH})$ without steatosis, with the highest GSK-3 $\beta$ inactivation because of Akt activation during hyperglycemia; mitochondrial function was not impaired, compared to the $18 \mathrm{~S}$ group. Euglycemic-18R showed neither GH nor steatosis, with intermediate GSK-3 $\beta$ activation and mitochondrial dysfunction. However, euglycemic-6R showed both $\mathrm{GH}$ and steatosis despite the highest GSK-3 $\beta$ activity and no molecular evidence of increased lipogenesis or decreased

${ }^{*}$ Corresponding author. Tel: +82-2-2030-7787; Fax: +82-2-20496192; E-mail: yunhee.noh@kku.ac.kr

${ }^{\#}$ These authors contributed equally to this work.

https://doi.org/10.5483/BMBRep.2020.53.2.114

Received 17 April 2019, Revised 7 May 2019, Accepted 9 June 2019

Keywords: Diabetes, Glycogenic hepatopathy, GSK-3ß, Mitochondrial function, Non-alcoholic hepatosteatosis
ApoB expression, where mitochondrial dysfunction was highest among the groups. In conclusion, heterogeneous liver histopathology developed in end-stage non-obese diabetic rats as the glycemic levels varied with differential diets, in which protein content in the diets as well as glycemic levels differentially influenced GSK-3 $\beta$ activity and mitochondrial function in insulin-deficient state. [BMB Reports 2020; 53(2): 100-105]

\section{INTRODUCTION}

Diabetes is commonly associated with liver disease, and the liver-related risk of mortality is significantly high in diabetes, compared to that in the general population (1).

Diabetic livers show heterogeneous histopathology, including hepatosteatosis, steatohepatitis, steatofibrosis, glycogenic hepatopathy $(\mathrm{GH}$; pathological overloading of glycogen within hepatocytes), and hepatosclerosis (perisinusoidal fibrosis) (2-4). However, it remains unclear whether diabetes per se (lack of insulin, but not compromised by obesity) does indeed affect the development of heterogeneity in liver histopathology, and what underlying molecular mechanisms are involved in this heterogeneity. Recently, renal histopathology has also been revealed to be heterogeneous in diabetes, where glycemic levels, age, systemic hypertension, and duration of diabetes were associated with heterogeneity (5-7). We hypothesized glycemic levels influence liver histopathology in insulindeficient diabetes, for euglycemia achieved by diet restriction in insulin-deficient diabetes increases autophagy and apoptosis in the liver tissue, as compared to hyperglycemia in insulindeficient diabetes or euglycemia in non-diabetes $(8,9)$.

Meanwhile, since diabetes is a disease that deteriorates over time and shows a wide-spectrum of glycemic levels $(10,11)$, a time-point in the course of diabetes and glycemic range was to 
be determined for the present study. Considering also current clinical situation where euglycemia is sometimes achieved by strict diet restriction but hyperglycemia generally prevails, we decided to investigate liver histopathology at the end-stage of diabetes with hyperglycemia or euglycemia, but without obesity. To mimic end-stage diabetes, in which loss of $\beta$-cell function and mass is significant (12), subtotal-pancreatectomy (subtotal-Px) was performed in rats.

In this background we aimed to see whether heterogeneity of liver histopathology can develop in diabetic rats fed differential diets to induce variation in glycemic levels as in real clinical settings, and to evaluate the underlying molecular mechanisms of heterogeneity.

Identification of glycemic and clinical factors associated with heterogeneity in diabetic kidney disease provides insight into diabetic nephropathy $(5,7)$. Likewise, we expect that our results would extend the current understanding of the relationship between diabetes and liver disease, particularly in diabetes that is not compromised by obesity, which is more common in Asian than in Western people.

\section{RESULTS}

\section{Liver histopathology is heterogeneous in diabetic rats fed differential diets}

First, we generated end-stage non-obese diabetic model rats by subtotal-Px, and subsequent ad libitum feeding of a standard chow (carbohydrate $62 \%$, protein $18 \%$, and lipid $20 \%$, by calories; more detailed information described in Table S1) for 7 weeks (diabetes induction period, Fig. 1A). During the induction period, all Px rats exhibited overt diabetic symptoms, such as fasting hyperglycemia and weight loss (Figs. 1B and C). The plasma insulin and C-peptide levels in all Px rats were significantly lower than in the sham-operated control $18 \mathrm{~S}$ rats, approximately $10-20 \%$ of the levels of the $18 \mathrm{~S}$ group (Table 1). Next, we divided the diabetic Px rats into three groups, which were fed differential diets for an additional 7 weeks (differential diet period) to modulate glycemic level: Px rats in the 18AL group were fed ad libitum the standard chow to maintain hyperglycemia, while the remaining $\mathrm{Px}$ rats were fed calorie-restricted diets of the standard chow (18R) or of a low-protein chow (6R; carbohydrate $74 \%$, protein $6 \%$, and lipid $20 \%$, by calories) to achieve euglycemia (Figs. 1A and B). The 6R group was designed to simulate a low-protein diet frequently prescribed for patients with diabetic nephropathy. The rats in the $18 \mathrm{~S}$ group were fed ad libitum the standard chow throughout the study, showing euglycemia and steady increase in body weight (Figs. $1 \mathrm{~A}-\mathrm{C}$ ). The rate of daily food intake in both $\mathrm{R}$ groups decreased to that in the $18 \mathrm{~S}$ group during the differential diet period, while the rate in the $18 \mathrm{AL}$ group was

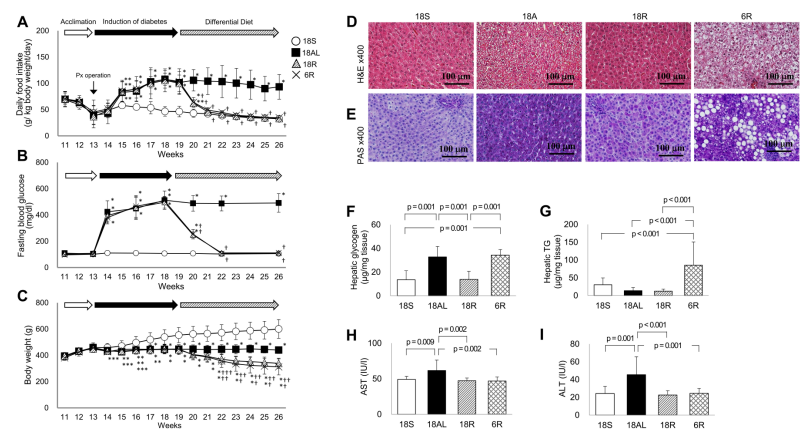

Fig. 1. Heterogeneity in liver histopathology, hepatic glycogen and TG contents, and serum hepatic enzymes when fed differential diets in end-stage non-obese model diabetic rats generated by subtotal-pancreatectomy. (A-C) Sequential changes in (A) the rate of daily food intake, (B) fasting blood glucose, and (C) body weight by the study schedule. (D, E) Liver histopathology demonstrated by (D) H\&E and (E) PAS staining. Hepatic content of (F) glycogen and (G) TG. Serum level of (H) AST and (I) ALT. Rats were treated as described in the Table 1 legend. 18S $(\bigcirc)$, 18AL $(\square), 18 R(\mathbb{Q})$, and $6 \mathrm{R}(\mathrm{X})$. Data are presented as the means $\pm \mathrm{SD}(\mathrm{n}=11$ per group). The data were analyzed by one-way ANOVA with Tukey's post hoc test. ${ }^{* * *} \mathrm{P}<0.05$, **P $<0.01$, ${ }^{*} \mathrm{P}<0.001$ vs 18S;

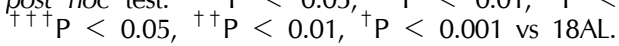

Table 1. Plasma insulin and C-peptide, and serum lipid profiles

\begin{tabular}{lccc}
\hline & $18 \mathrm{~S}$ & $18 \mathrm{AL}$ & \multicolumn{1}{c}{$18 \mathrm{R}$} \\
\hline Insulin $(\mu \mathrm{U} / \mathrm{L})$ & $39.8 \pm 22.6$ & $4.7 \pm 2.4^{*}$ & $3.3 \pm 2.0^{*}$ \\
C-peptide $(\mathrm{nmol} / \mathrm{mL})$ & $1.3 \pm 0.3$ & $0.3 \pm 0.1^{*}$ & $0.3 \pm 0.1^{*}$ \\
Total cholesterol $(\mathrm{mg} / \mathrm{dL})$ & $218.6 \pm 66.9$ & $183.3 \pm 96.5$ & $184.5 \pm 70.5$ \\
Triglyceride $(\mathrm{mg} / \mathrm{dL})$ & $142.3 \pm 34.8$ & $100.8 \pm 26.3^{* *}$ & $64.3 \pm 16.9^{*}$ \\
HDL-cholesterol $(\mathrm{mg} / \mathrm{dL})$ & $86.0 \pm 19.5$ & $47.1 \pm 9.2^{*}$ & $51.1 \pm 8.7^{*}$ \\
\hline
\end{tabular}

$18 \mathrm{~S}$, sham-operated control rats on a standard chow ad libitum (protein 18, carbohydrate 62, lipid 20 kcal\%), showing euglycemia throughout the study; end-stage non-obese diabetic model rats generated by subtotal-pancreatectomy on the standard chow ad libitum to maintain hyperglycemia $(18 \mathrm{AL})$, on a calorie-restricted diet to achieve euglycemia with the standard chow (18R), or with a low-protein chow (6R; protein 6 , carbohydrate 74 , lipid $20 \mathrm{kcal} \%$ ) during the differential diet period [the rate of food intake ( $/ \mathrm{kg}$ of body weight/day) of both $\mathrm{R}$ groups was the same as that in the $18 \mathrm{~S}$ group]. Data are presented as the means $\pm \mathrm{SD}$. ( $\mathrm{n}=11$ per group). The data were analyzed by one-way ANOVA with Tukey's post hoc test; $* \mathrm{P}<0.001,{ }^{* * P}<0.01$, vs $18 \mathrm{~S} ;{ }^{\dagger} \mathrm{P}<0.05$, vs $18 \mathrm{AL}$. 
maintained at the same level as in the diabetes induction period, showing 2.7-fold higher than that in the remaining groups at euthanization (Fig. 1A). The body weights at euthanization were significantly lower in both $\mathrm{R}$ groups than in the $18 \mathrm{~S}$ or the $18 \mathrm{AL}$ group (Fig. 1C), although the levels of plasma insulin and C-peptide among all Px groups did not differ (Table 1).

The results of H\&E staining, PAS staining, and hepatic glycogen and triglycerides (TG) content (Figs. 1D-G) revealed the livers of end stage non-obese diabetic rats showed heterogeneous histopathology after overnight fasting, which depended on not only the glycemic levels but also the protein content in the diet: the $18 \mathrm{AL}$ and $6 \mathrm{R}$ groups showed high accumulation of glycogen compared to the $18 \mathrm{~S}$ and $18 \mathrm{R}$ groups (Figs. 1E and F), and 6R exhibited steatosis (Figs. 1D, E, and G).

Serum TG and HDL-C levels were significantly lower in all Px groups than in the $18 \mathrm{~S}$ group (Table 1 ). However, serum AST and ALT levels were significantly higher only in the 18AL group than in the other groups (Figs. $1 \mathrm{H}$ and $\mathrm{I}$ ).

\section{Ultrastructural liver images were heterogeneous in diabetic rats, which was partially associated with the activities of regulatory factors for hepatic glycogen or lipid synthesis}

To confirm the heterogeneity at ultrastructural level we performed transmission-electron microscopy (TEM; Fig. 2A): the hepatocytes from the hyperglycemic-18AL group contained many glycogen particles but few autophagosomes, while those from the euglycemic-diabetic $6 \mathrm{R}$ group showed

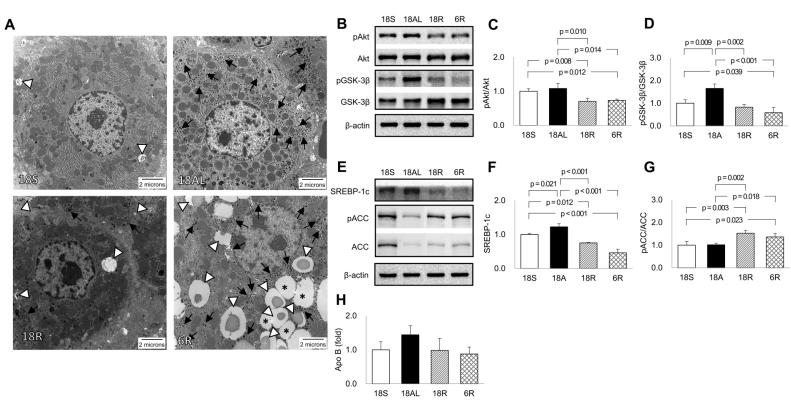

Fig. 2. Heterogeneity in the ultrastructure of hepatocytes and activities of regulatory factors for hepatic glycogen or lipid synthesis. (A) Liver TEM images. (B-D) Molecular variations in the regulatory factors for glycogen synthesis determined by (B) western blotting for Akt and GSK-3 $\beta$, and by densitometry for (C)

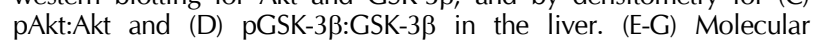
variations in the regulatory factors for lipid synthesis determined by (E) western blotting for SREBP-1C and ACC, and by densitometry for (F) SREBP-1C and (G) pACC:ACC in the liver. $(\mathrm{H})$ mRNA expression for apolipoprotein $B$ in the liver determined by $\Delta \mathrm{Ct}$ values after real-time RT-PCR. Rats were treated as described in the Table 1 legend. Data are presented as the means \pm SD ( $n$ $=5$ per group). The data were analyzed by one-way ANOVA with Tukey's post hoc test. Arrowheads, autophagosomes; Arrows, glycogen particles; Asterisks, lipid droplets. many glycogen particles, autophagosomes, and large lipid droplets. The hepatocytes from another euglycemic-diabetic $18 \mathrm{R}$ group showed some autophagosomes but little glycogen particles, while those from euglycemic non-diabetic $18 \mathrm{~S}$ rats showed few autophagosomes and no glycogen particles.

To explain the heterogeneous histopathology in the livers at molecular level, we examined the regulatory factors of glycogen or lipid synthesis in the liver tissue by western blotting. First, we assessed the regulation of glycogen synthesis (Figs. 2B-D): the degree of Akt activation (a major insulin signalling modality) was similar in hyperglycemic-18AL and euglycemic non-diabetic $18 \mathrm{~S}$ groups, while the degree in euglycemic-diabetic18R and $6 \mathrm{R}$ groups decreased significantly by $\sim 30 \%$, compared to that in the $18 \mathrm{~S}$ or $18 \mathrm{AL}$ groups. Glycogen synthase kinase-3 $\beta$ (GSK-3 $\beta$ ) phosphorylation, a downstream signalling of pAkt, which leads to glycogen synthase activation, was highest in the 18AL group, and lowest in the $6 \mathrm{R}$ group among all experimental groups. However, the level was similar in the $18 \mathrm{R}$ and $18 \mathrm{~S}$ groups.

Next, we assessed the regulation of lipid synthesis (Figs. $2 \mathrm{E}-\mathrm{G})$ : the level of sterol regulatory element-binding protein-1c (SREBP-1C; transcription factor for fatty acid synthesis, whose expression is upregulated by insulin) was highest in the hyperglycemic-18AL group among all experimental groups, 1.2-fold higher than in the non-diabetic $18 \mathrm{~S}$ group $(\mathrm{P}=$ $0.021)$. In contrast, the levels in both euglycemic-diabetic $18 \mathrm{R}$ and $6 \mathrm{R}$ groups significantly decreased by 25 and $54 \%$, respectively, compared to that in the $18 \mathrm{~S}$ groups. Acetyl-CoA carboxylase (ACC) is the rate-limiting enzyme in fatty acid synthesis, whose phosphorylation (an inactivation modality against ACC) levels in the $18 \mathrm{R}$ and $6 \mathrm{R}$ groups increased

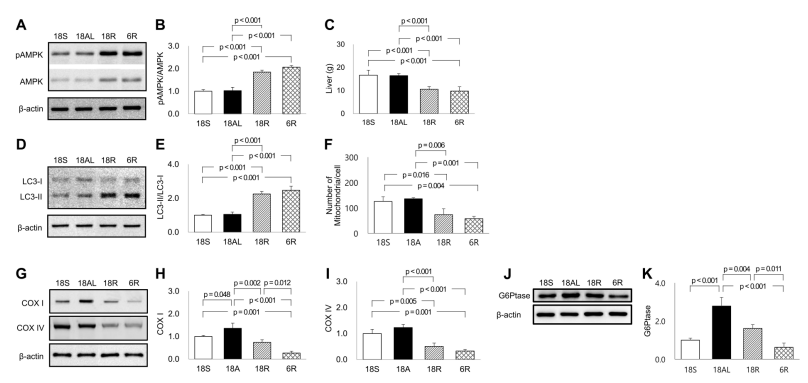

Fig. 3. Molecular mechanisms of differential liver weights and nutrient accumulation in the livers of end-stage non-obese model diabetic rats on differential diets. (A-E) AMPK activation by (A) western blotting and (B) densitometry, (C) liver weight, and LC3-II:LC3-I determined by (D) western blotting and (E) densitometry. (F-I) Mitochondrial function in terms of $(\mathrm{F})$ mitochondrial number, and the levels of COX I and COX IV determined by (G) western blotting, and densitometry for $(\mathrm{H})$ COX I and (I) COX IV, respectively. (J, K) The level of G6Ptase determined by (J) western blotting and $(K)$ densitometry. Rats were treated as described in the Table 1 legend. Data are presented as the means \pm SD (liver weight, $\mathrm{n}=11$ per group; western blotting $\mathrm{n}=5$ per group). The data were analyzed by one-way ANOVA with Tukey's post hoc test. 
significantly by $1.5-$ and 1.4 -fold, respectively, compared to those in the $18 \mathrm{~S}$ and $18 \mathrm{AL}$ groups, respectively. However, the phosphorylation levels of ACC did not differ between the $18 \mathrm{~S}$ and $18 \mathrm{AL}$ groups.

To examine whether lipid accumulation in the livers of rats in the 6R group was attributed to the lack of ApoB (a major constituent apolipoprotein in VLDL) gene expression, we performed real-time RT-PCR analysis of hepatic ApoB mRNA. However, the mRNA level did not differ significantly among groups, as determined by comparing the amplification products by calculating the $\Delta \mathrm{Ct}$ values (Fig. $2 \mathrm{H}$ ).

\section{Heterogeneous liver histopathology in diabetic rats is associated with mitochondrial dysfunction}

To explain the discrepancies between the molecular and histopathological findings observed in the $6 \mathrm{R}$ group, in which glycogen and lipid accumulated in hepatocytes despite the decreased signals for glycogenesis and lipogenesis (compared to those in the $18 \mathrm{~S}$ or $18 \mathrm{AL}$ group), we examined AMPK phosphorylation (a major intracellular energy sensing modality) by western blotting (Figs. 3A and B). Both euglycemic-diabetic $18 \mathrm{R}$ and $6 \mathrm{R}$ groups showed an approximately two-fold increase in AMPK activation compared to the $18 \mathrm{~S}$ and $18 \mathrm{AL}$ groups ( $\mathrm{P}<0.001$ for all), where energy sources such as glycogen and TG were abundant in the $6 \mathrm{R}$ group. The level of AMPK activation in the hyperglycemic-18AL group was similar to that in the euglycemic non-diabetic $18 \mathrm{~S}$ group.

When we examined the LC3-II to LC3-I ratio, an index of autophagy, to evaluate the downstream regulation of AMPK, the ratio increased by more than two-fold in euglycemicdiabetic rats in both $\mathrm{R}$ groups, compared to that in $18 \mathrm{~S}$ or 18AL rats (Figs. 3D and E). However, this ratio did not differ between the $18 \mathrm{~S}$ and $18 \mathrm{AL}$ groups. Consistent with these findings, the liver weight in the $18 \mathrm{R}$ or $6 \mathrm{R}$ group, which showed increased AMPK activity and autophagy, decreased by $\sim 40 \%$ compared to that in the $18 \mathrm{~S}$ or $18 \mathrm{AL}$ group (Figs. $2 \mathrm{~A}$ and $3 \mathrm{~A}-\mathrm{E})$. However, the liver weight in the $18 \mathrm{AL}$ group did not differ significantly from that in the $18 \mathrm{~S}$ group.

We next examined hepatic mitochondrial function in terms of protein expression levels of electron transport chain complexes (COX) by western blotting (Figs. 3G-I), for we had observed that the mitochondrial number per hepatocyte decreased in the $18 \mathrm{R}$ or $6 \mathrm{R}$ rats compared to that in the $18 \mathrm{~S}$ or $18 \mathrm{AL}$ rats (Figs. $2 \mathrm{~A}$ and $3 \mathrm{~F}$ ). The mitochondrial COX I level was highest in the 18AL group among all groups. However, the level of COX I in the 6R group decreased by 74 and $65 \%$, respectively, compared to that in the $18 \mathrm{~S}$ or $18 \mathrm{R}$ group. COX IV level significantly decreased in both $\mathrm{R}$ groups by 50 and $67 \%$, respectively, compared to that in the $18 \mathrm{~S}$ group. The level of COX IV did not differ between the $18 \mathrm{~S}$ and 18AL groups.

Finally, we examined the level of hepatic G6Ptase by western blotting (Figs. 3J and K) to determine if the glycogen accumulation is associated with the level of G6Ptase, the last enzyme in the glycogenolysis pathway. The G6Ptase level in the $6 R$ rats significantly decreased by 77 and $61 \%$, respectively, compared to that in the $18 \mathrm{AL}$ or $18 \mathrm{R}$ rats.

\section{DISCUSSION}

In the present study, we demonstrated that heterogeneous histopathology developed in the livers of end-stage non-obese diabetic model rats as the glycemic level varied with differential diets, in which protein content in the diets, as well as glycemic levels, differentially influenced GSK-3 $\beta$ activity and mitochondrial function in insulin-deficient state. The heterogeneity ranged from apparently normal histology to $\mathrm{GH}$ and steatosis, although the insulin and C-peptide levels did not differ among the diabetic rats.

Several studies reported $\mathrm{GH}$ (accompanied by elevated liver transaminases and hepatomegaly, but rarely hepatic steatosis) as an under-recognized hepatic complication among type 1 and 2 diabetic patients who received insulin treatment, but had poor long-term glycemic control $(3,13,14)$. Interestingly, our hyperglycemic insulin-deficient $18 \mathrm{AL}$ group showed the same phenotypes, including $\mathrm{GH}$ with elevated liver transaminases and the highest liver weight among Px diabetic rat groups, but not accompanied by hepatic steatosis (Figs. 1D-I and 2A). The molecular mechanism of glycogen accumulation in the liver of diabetic patients is not well understood, for the insulin signaling that potently promotes glycogen synthesis is reduced in diabetes. The proposed mechanism for $\mathrm{GH}$ in diabetes is that the exogenous insulin administered to treat diabetes, and the increased glucose 6 phosphate level in hepatocytes resulting from hyperglycemia, play roles in upregulating hepatic glycogen synthesis (14-16). However, our results clearly demonstrated that $\mathrm{GH}$ developed in the insulin deficient-state alone, without the additional action of exogenous insulin, when hyperglycemia was prolonged by an ad libitum diet. Akt was activated during hyperglycemia independently of insulin in the $18 \mathrm{AL}$ rats, as described previously $(8,9,17,18)$. Therefore, we propose that one of the mechanisms of $\mathrm{GH}$ in diabetes involves upregulated glycogen synthesis in hepatocytes because of increased GSK-3 $\beta$ phosphorylation by the activated Akt during hyperglycemia, although the G6Ptase level was greatly upregulated; phosphorylated GSK-3 $\beta$ lost GSK-3 $\beta^{\prime}$ 's inhibitory function over glycogen synthase, resulting in glycogen accumulation in the $18 \mathrm{AL}$ group (Figs. 2A-D).

Meanwhile, the $18 \mathrm{R}$ group maintained an apparently normal liver histology, neither $\mathrm{GH}$ nor steatosis, showing a similar level of GSK-3 $\beta$ phosphorylation compared to the $18 \mathrm{~S}$ group (Figs. 1D-G and 2A, B, D), indicating that glycogen synthesis was similarly inhibited in the $18 \mathrm{~S}$ and $18 \mathrm{R}$ groups. In contrast to the normal liver histology observed in the $18 \mathrm{R}$ group, the other euglycemic-diabetic rats in the $6 \mathrm{R}$ group showed $\mathrm{GH}$ and steatosis in the presence of excessive autophagy. Despite the difference in histopathology, the liver weights in both $\mathrm{R}$ 
groups decreased to the level of those in non-diabetic rats following long-term starvation at the near-death point (19), through excessive autophagy induced by decreased Akt, and increased AMPK activation during euglycemia in the insulindeficient state (Figs. 2A-C and 3A-E). Although the level of GSK-3 $\beta$ phosphorylation was lowest in the $6 \mathrm{R}$ group, we paradoxically observed $\mathrm{GH}$ in the $6 \mathrm{R}$ group; we speculated that the lowest level of G6Ptase in the 6R group compared to the other Px groups contributed to inhibit glycogenolysis and to result in glycogen accumulation in the $6 \mathrm{R}$ group (Figs. 3J and K).

The similar levels of ApoB expression among all experimental groups suggest that VLDL synthesis for disposing TG into general circulation was not impaired, even in $6 \mathrm{R}$ rats (Fig. $2 \mathrm{H}$ ). Neither the size of epididymal fat pads $(12 \%$ of that in $18 \mathrm{~S}$ rats, data not shown) nor euglycemia in the $6 \mathrm{R}$ rats supports the possibility of increased influx of free fatty acids (FFA) from systemic adipose tissue or increased uptake of glucose into the hepatocytes to explain their hepatic steatosis or $\mathrm{GH}$.

However, mitochondrial dysfunction, particularly the lowest level of COX I, together with the decreased COX IV and mitochondrial number per hepatocyte in the $6 R$ group, compared to those in the other groups, suggests a mechanism for glycogen and TG accumulation in hepatocytes, despite AMPK activation. Autophagy is also upregulated by amino acid depletion and decreased insulin signaling (20); we speculate that isocaloric control of diet with low-protein in the $6 R$ rats may accelerate autophagy, leading to engulfment of organelles, including mitochondria, to meet a large deficit of amino acid requirements compared to that in the $18 \mathrm{R}$ rats. Since the mitochondria had been consumed by excessive autophagy, the energy sources within the hepatocytes of $6 R$ rats could not be used (oxidized), and thus accumulated. Interestingly, lipid accumulation in the liver has been reported in healthy lean elderly patients with mitochondrial dysfunction (21), suggesting that energy sources, unused because of mitochondrial dysfunction, accumulate intracellularly. A generally accepted pathophysiology of hepatic steatosis in diabetes associated with obesity is that increased influx of FFA from systemic adipose tissue promotes lipogenesis and TG accumulation in the liver $(2,22,23)$; however, studies have shown that mitochondrial dysfunction precedes insulin resistance and hepatic steatosis $(24,25)$.

Finally, we propose that hyperglycemia and insulin deficiency per se may not cause mitochondrial dysfunction; Franko et al. reported elevated COX I and COX II levels in hyperglycemic diabetic rats with insulin deficiency (26). Consistently, our hyperglycemic-18AL rats showed elevated COX I level and similar levels of COX IV and mitochondrial number per hepatocyte compared to those in the $18 \mathrm{~S}$ rats (Figs. 3F-I). We speculated that since the maintained mitochondrial function in 18AL rats enabled $\beta$-oxidation of fatty acids at normal velocity, steatosis was not observed, though lipogenic signals (SREBP-1C and ACC) increased by Akt activation in the $18 \mathrm{AL}$ group compared to those in both $\mathrm{R}$ groups (Figs. 1D, E, G and 2A, E-G).

Our study had some limitations. First, we used end-stage non-obese diabetic model rats to observe diabetic liver histopathology, so our data may not be directly related to the hepatic histopathology in diabetes with obesity and insulin resistance. However, insulin resistance ultimately results in impaired insulin signaling, and thus our data may be a useful reference for interpreting the histopathology in diabetes with insulin resistance. Second, in diabetic liver histopathology, we did not observe the development of hepatic fibrosis by Masson trichrome staining (data not shown). Further studies are needed to determine whether a 7-week differential diet period is too short to induce hepatic fibrosis, or a new inducing factor rather than our differential diets is required to induce hepatic fibrosis in diabetes.

In summary, as observed previously in diabetic kidneys, insulin-deficient diabetes induced heterogeneous histopathology in the liver, where $\mathrm{GH}$ was induced during hyperglycemia, or normal histology but with a detrimentally decreased liver weight during euglycemia; however, when euglycemia was achieved using a low-protein diet control, $\mathrm{GH}$ and steatosis and a detrimental reduction of liver weight occurred. The most important molecular mechanisms in the heterogeneity of liver histopathology in insulin deficiency included varying degrees of GSK-3 $\beta$ phosphorylation and mitochondrial dysfunction, which were regulated according to Akt and AMPK activities and autophagy levels, initially triggered by energy and/or amino acid requirements in hepatocytes during insulin deficiency.

In conclusion, insulin deficiency alone is not considered a sufficient factor to induce heterogeneous liver histopathology; rather, nutrient levels in the blood influenced by diets are simultaneously important factors. To understand the heterogeneous histopathology in the diabetic liver, we should consider that the liver is a dynamic organ that timely compensates for unmet metabolic needs in the environment of lacking the anabolic signal, insulin.

\section{MATERIALS AND METHODS}

The detailed methods are described in the "Supplementary Materials and Methods".

\section{CONFLICTS OF INTEREST}

The authors have no conflicting interests.

\section{REFERENCES}

1. Adams LA, Harmsen S, St Sauver JL et al (2010) Nonalcoholic fatty liver disease increases risk of death among patients with diabetes: a community-based cohort study. Am J Gastroenterol 105, 1567-1573

2. Roden M (2006) Mechanisms of Disease: hepatic steatosis in type 2 diabetes-pathogenesis and clinical relevance. 
Nat Clin Pract Endocrinol Metab 2, 335-348

3. Torbenson $M$, Chen YY, Brunt $E$ et al (2006) Cummings, O.W.; Gottfried, M.; Jakate, S.; Liu. Y.C.; Yeh, M.M.; Ferrell, L. Glycogenic hepatopathy: an underrecognized hepatic complication of diabetes mellitus. Am J Surg Pathol 30, 508-513

4. Harrison SA. Brunt EM. Goodman ZD et al (2006) Diabetic hepatosclerosis: diabetic microangiopathy of the liver. Arch Pathol Lab Med 130, 27-32

5. Fioretto $P$, Mauer M, Brocco E et al (1996) Patterns of renal injury in NIDDM patients with microalbuminuria. Diabetologia 39, 1569-1576

6. Ekinci El, Jerums G, Skene A et al (2013) Renal structure in normoalbuminuric and albuminuric patients with type 2 diabetes and impaired renal function. Diabetes Care 36, 3620-3626

7. Sharma SG, Bomback AS, Radhakrishnan J et al (2013) The modern spectrum of renal biopsy findings in patients with diabetes. Clin J Am Soc Nephrol 8, 1718-1724

8. Lee JH, Lee JH, Jin M et al (2014) Diet control to achieve euglycemia induces significant loss of heart and liver weight via increased autophagy compared with ad libitum diet in diabetic rats. Exp Mol Med 46, e111

9. Lee JH, Choi SB, Jin M et al (2015) Euglycemia in Diabetic Rats Leads to Reduced Liver Weight via Increased Autophagy and Apoptosis through Increased AMPK and Caspase-3 and Decreased mTOR Activities. J Diabetes Res 2015, 497431

10. Prentki $M$ and Nolan CJ (2006) Islet beta cell failure in type 2 diabetes. J Clin Invest 116, 1802-1812

11. Rahier J, Guiot Y, Goebbels RM et al (2008) Pancreatic beta-cell mass in European subjects with type 2 diabetes. Diabetes Obes Metab 10, 32-42

12. Halban PA, Polonsky KS, Bowden DW et al (2014) $\beta$-cell failure in type 2 diabetes: postulated mechanisms and prospects for prevention and treatment. Diabetes Care 37, 1751-1758

13. Nakamuta $M$, Ohashi $M$, Goto K et al (1993) Diabetes mellitus-associated glycogen storage hepatomegaly: report of a case and review of the Japanese literature. Fukuoka Igaku Zasshi 84, 354-358

14. Chatila R and West AB (1993) Hepatomegaly and abnormal liver tests due to glycogenosis in adults with diabetes. Medicine (Baltimore) 75, 327-333

15. Van Steenbergen W and Lanckmans S (1995) Liver disturbances in obesity and diabetes mellitus. Int J Obes Relat Metab Disord 19, S27-S36

16. Munns CF, McCrossin RB, Thomsett MJ et al (2000) Hepatic glycogenosis: reversible hepatomegaly in type 1 diabetes. J Paediatr Child Health 36, 449-452

17. Li SY, Fang CX, Aberle NS 2nd et al (2005) Inhibition of $\mathrm{PI}-3$ kinase/Akt/mTOR, but not calcineurin signaling, reverses insulin-like growth factor I-induced protection against glucose toxicity in cardiomyocyte contractile function. J Endocrinol 186, 491-503

18. Mariappan MM, Shetty M, Sataranatarajan K et al (2008) Glycogen synthase kinase 3beta is a novel regulator of high glucose- and high insulin-induced extracellular matrix protein synthesis in renal proximal tubular epithelial cells. J Biol Chem 283, 30566-30575

19. Goodman MN and Ruderman NB (1980) Starvation in the rat. I. Effect of age and obesity on organ weights, RNA, DNA, and protein. Am J Physiol 239, E269-E276

20. Singh R and Cuervo AM (2011) Autophagy in the cellular energetic balance. Cell Metab 13, 495-504

21. Petersen KF, Befroy D, Dufour S et al (2003) Mitochondrial dysfunction in the elderly: possible role in insulin resistance. Science 300,1140-1142

22. Smith BW and Adams LA (2011) Nonalcoholic fatty liver disease and diabetes mellitus: pathogenesis and treatment. Nat Rev Endocrinol 7, 456-465

23. Lewis GF, Carpentier A, Adeli K et al (2002) Disordered fat storage and mobilization in the pathogenesis of insulin resistance and type 2 diabetes. Endocr Rev 23, 201-229

24. Ibdah JA, Perlegas $P$, Zhao $Y$ et al (2005) Mice heterozygous for a defect in mitochondrial trifunctional protein develop hepatic steatosis and insulin resistance. Gastroenterology 128, 1381-1390

25. Rector RS, Thyfault JP, Uptergrove GM et al (2010) Mitochondrial dysfunction precedes insulin resistance and hepatic steatosis and contributes to the natural history of non-alcoholic fatty liver disease in an obese rodent model. J Hepatol 52, 727-736

26. Franko A, von Kleist-Retzow JC, Neschen S et al (2014) Liver adapts mitochondrial function to insulin resistant and diabetic states in mice. J Hepatol 60, 816-823 